nium, from the reaction $U^{238}(d, n) 93^{239}$. It is impossible to deduce from the absorption curve the relative intensities of the new 93 and of $93^{239}$, since the initial parts of the individual absorption curves of these two activities might well be nearly identical. The rate of decay of the high energy beta-particles (0.5-1 Mev) and gamma-rays from the 93 of uranium plus deuterons was determined. This gave a half-life of about 2 days for the new 93 . This activity is probably to be assigned to $93^{238}, 93^{238}$, or $93^{235}$ formed in the reaction $\mathrm{U}^{238}(d, 2 n) 93^{238}, \mathrm{U}^{235}(d, n) 93^{236}$, or $\mathrm{U}^{235}(d, 2 n) 93^{235}$, respectively.

The growth of alpha-particles, which might be due to the element 94 daughter of the 2-day 93, was then looked for. We did observe the growth of alpha-particles in the very carefully purified, as well as in the semi-purified 93 fractions, and the growth curves indicate a half-life of roughly 2 days for the parent of the alpha-emitter. The final alpha-particle count amounts to several hundred counts per minute for a bombardment of 200 microamperehours. This work was done with a proportional type counter. We plan to re-determine the alpha-particle growth curve more accurately using an ionization chamber and linear amplifier with the help of a magnetic field to bend out the very strong beta-particle background. The alphaparticles have a range of approximately $3.9 \mathrm{~cm}$ in air.

This alpha-activity is chemically separable from uranium and 93. The chemical experiments so far indicate a similarity to thorium and the activity has not yet been separated from thorium. More chemical experiments definitely must be performed before it can be regarded as proved that the alpha-particles are due to an isotope of element 94 .

* This letter was received for publication on the date indicated but was voluntarily withheld from publication until the end of the war.

\section{Radioactive Element 94 from Deuterons on Uranium}

G. T. Seaborg, A. C. Wahl, and J. W. Kennedy Department of Chemistry, Radiation Laboratory, Department of Physics University of California, Berkeley, California March 7, 1941*

$\mathbf{W}$

E should like to report a few more results which we have found regarding the element 94 alpharadioactivity formed in the $16-\mathrm{Mev}$ deuteron bombardment of uranium. We sent a first report $t^{1}$ of this work in a Letter to the Editor of January 28, 1941. We have in the meantime performed more experiments in order to study the chemical behavior of this alpha-radioactive isotope. The radioactivity can be precipitated, in what is probably the +4 valence state, as a fluoride or iodate by using a rare earth or thorium as carrier material and as a peroxyhydrate by using thorium as carrier material. However, in the presence of the extremely strong oxidizing agent persulfate ion $\left(\mathrm{S}_{2} \mathrm{O}_{8}^{-}\right)$, plus $\mathrm{Ag}$ as a catalyst, this radioactive isotope is oxidized to a higher valence state which does not precipitate as a fluoride. The oxidizing agent bromate ion $\left(\mathrm{BrO}_{3}^{-}\right)$is not sufficiently powerful to oxidize it to this higher valence state and hence the radioactivity comes down as a fluoride even in the presence of bromate ion. With the help of persulfate ion it has been possible to separate quantitatively this radioactivity from thorium, by using the beta-active $\mathrm{UX}_{1}$ as an indicator for thorium. These experiments make it extremely probable that this alpha-radioactivity is due to an isotope of element 94 . The experiments are being continued.

* This letter was received for publication on the date indicated but was voluntarily withheld from publication until the end of the war. ${ }^{1}$ G. T. Seaborg, E. M. McMillan, J. W. Kennedy and A. C. Wahl Phys. Rev. 69, 366 (1946).

\section{Search for Spontaneous Fission in $\mathbf{9 4 ^ { 2 3 9 }}$ \\ JOSEPH W. KENNEDY AND ARTHUR C. WAHL. Berkeley, California December 4, 1941*}

$T$ IS report describes the experimental procedure and gives the results obtained in our search for spon taneous fission in $94^{239}$. The observations were made on a 3.5-microgram sample of $94^{239}$, the preparation of which was described in a report of July 24,1941 , from this laboratory. This sample, when placed on one electrode of an ionization chamber connected to a linear amplifier adjusted to record counts due to fissions, gave zero counts in 139 hours. In another, independent experiment, done in a similar manner with entirely different apparatus, this sample gave zero counts in 209 hours. These experiments set a lower limit of the order of $10^{14}$ years for the "half-life" of $94^{239}$ with respect to spontaneous fission. These results show that it is probable that the half-life for spontaneous fission of $94^{239}$ is as long or longer than that of $\mathrm{U}^{235}$, assuming the British results (40 spontaneous fissions per min. per $\mathrm{g}$ of pure $\mathrm{U}^{235}$ ).

The details follow: The 3.5-microgram sample of $94^{239}$ was mounted on a platinum-coated copper disk, along with its cerium fluoride carrier in which the thickness amounted to $\sim 0.3 \mathrm{mg}$ per $\mathrm{cm}^{2}$. All the measurements described herein were made with shallow ionization chambers so constructed that this disk could be inserted to serve as one of the electrodes. Consideration of this geometry indicates that ionizing particles emitted from a sample on the disk could be counted with an efficiency of about 45 percent; tests of the alpha-particle counting rate with a thin, weighed uranium sample on a similar disk confirmed this estimate when the gain of the linear amplifier used was set sufficiently high. Since in each fission process two ionizing particles are emitted in opposite directions, an upper limit of about 90 percent efficiency for fission counting is set by the geometrical arrangement. Actually, because of the fluctuating background ionization due to alphaparticles from the sample, it was expedient to adjust the amplifier gain in such a way that a slightly lower counting efficiency was obtained. With the use of a neutron source and a thin uranium sample mounted like the $94^{239}$ sample, two independent calibrations of this efficiency were made, as follows: (1) oscillographic observation of pulses showed the fraction of fission pulses too small to actuate the thryatron recording circuit; (2) the recorded fission rate was compared with the expected rate calculated from the weight of the thin uranium sample, the slow neutron fission cross 
section of uranium, and the density of slow neutrons as determined with an indium detector.

Both these calibrations gave the result that about 70 percent of the fissions were being recorded under the operating conditions of our experiments. This slight reduction in counting efficiency is no doubt to be attributed to discrimination against those fission fragments which traverse only a corner of the ionization chamber. It is of course apparent that high precision in the evaluation of this percentage efficiency is not required for our interpretation of the final result; however, the effect of small variations ( \pm 10 percent) in amplifier gain on this efficiency calibration was investigated and found negligible, showing that the working region approximated a plateau.

Two separate linear amplifier and ionization chamber outfits were used for two independent series of spontaneous fission experiments. At frequent intervals during all measurements the over-all gain of the amplifier was checked by the use of a pulse generator and voltage divider box or by re-testing the efficiency of counting slow-neutron induced fissions in uranium.

The series of measurements with one amplifier and chamber gave zero spontaneous fission counts in 139 hours of operation. With the other amplifier set-up, 209 hours of observation gave zero counts. These results enable us to say something about the spontaneous fission disintegration constant of $94^{239}$, or, more conveniently, about the halflife for spontaneous fission corresponding to this disintegration constant. In the two experiments taken together zero counts were obtained in 348 hours. From the weight of the sample ( 3.5 micrograms) and the efficiency of the apparatus for counting fissions (70 percent), one can calculate that for a half-life of $10^{13}$ years the mean time between counts would be about 20 hours and for a half-life of $10^{14}$ years the mean time between counts would be about 200 hours. The probability of obtaining zero counts in 348 hours is about $10^{-8}$ for 20 hours mean time between counts, and about 0.2 for 200 hours mean time between counts. Therefore, it seems fairly safe to say that the halflife of $94^{239}$ for spontaneous fission is of the order of $10^{14}$ years or greater.

* This letter was received for publication on the date indicated but was voluntarily withheld from publication until the end of the war.

\section{Determination of Absolute Neutron Intensities}

R. D. O'Neal and G. ScharfF-Goldhaber

Department of Physics, University of Illinois, Urbana, Illinois February 24, 1942*

$\mathrm{W}^{\mathrm{s}}$ E have developed a method of measuring the number of neutrons emitted from a given source and used it to determine the neutron intensity of a laboratory standard consisting of $100 \mathrm{mg}$ of radium surrounded by $85 \mathrm{~g}$ of beryllium metal. We made use of the main principle of Amaldi and Fermi's well-known method, which consists in slowing down all the neutrons emitted from the source before they are counted. However, we avoided the use of a number of constants and some difficult approximations, which appeared in the original form of this method.

Our procedure consisted essentially of two steps. (a) Determination of the ratio $R$ of the number of neutrons activating a $\mathrm{MnSO}_{4}$ solution ${ }^{1}$ with and without the presence of an absorber (consisting of finely powdered manganese).The volume of the solution was chosen so large that 1 percent of the neutrons was allowed to escape. After irradiation the solution was thoroughly stirred and the decay of its radioactivity observed by means of a large thin-walled Geiger counter (initial activity without absorber 1110 counts/min. above the background of 200 counts/min., with absorber 850 counts/min.).

(b) Determination of the number of neutrons $\left(N_{a}\right)$ captured by the absorber per unit time.-After irradiation a fraction of the well-mixed $\mathrm{Mn}$ powder was filled into paper shells. These "thick" samples were placed alternately around a thin-walled Geiger counter and their initial activities were determined ( 6185 counts $/ \mathrm{min}$.).

In order to determine the number of disintegrations per gram of manganese (specific activity), we irradiated some manganese powder with neutrons from a very strong source and filled a known amount of it into a paper shell as before. With another part we prepared a thin layer $\left(16 \mathrm{mg} / \mathrm{cm}^{2}\right.$ ) between two pieces of scotch tape, which was placed in a defined position relative to the counter. A similar layer, made of a known amount of fine uranium metal powder, was placed in the same position for standardization. For the calculation of the specific activity of the managanese samples the number of $\mathrm{UX}_{2}$ nuclei decaying per sec. per gram of uranium was assumed to be $12220 .^{2}$ A correction of 2.5 percent was found to be necessary to allow for the slightly stronger absorption of the $\mathrm{UX}_{2}$ beta-rays in the glass wall of the Geiger counter and in the scotch tape. By multiplying the specific activity of the manganese absorber with the total weight of the absorber $(52.3 \mathrm{~g})$, we obtained $N_{a}$ and $N=N_{a} /(1-R)$ where $N$ is the total number of neutrons emitted from our source.

The result was $N=(8.6 \pm 0.8) \times 10^{4}$ neutrons per sec. The error was calculated by adding up all standard deviations from the mean of the several measurements, which were made for each individual quantity.

The intensity of this laboratory standard has been compared with that of a Ra- $\alpha$-Be source (see following letter by Gamertsfelder and Goldhaber).

* This letter was received for publication on the date indicated but was voluntarily withheld from publication until the end of the war. ${ }^{1}$ The method of "physical integration" was first described by H. L. Anderson, E. Fermi, and L. Szilard, Phys. Rev. 56, 284 (1939) 2 Calculated from data given by A. F. Kovarik and N. J. Adams, Jr.,
J. App. Phys. 12, 296 (1941), and by A. O. Nier, Phys. Rev. 55, 150 (1939).

\section{A Reproducible Neutron Standard}

\author{
G. R. Gamertsfelder AND M. Goldhaber
} Department of Physics, University of Illinois, Urbana, Illinois February 24, 1942*

$F^{\circ}$ OR some time a need has been felt for a simple, reproducible standard of neutron intensity. It is known that the most commonly used neutron sources, those obtained by mixing $\mathrm{Rn}$ or $\mathrm{Ra}$ with $\mathrm{Be}$ powder, are not reproducible. ${ }^{1}$ By contrast it would appear that a photoneutron source, obtained by irradiating $\mathrm{Be}$ with $\mathrm{Ra} \gamma$ rays under exactly defined geometrical conditions, could 